\title{
In Vitro Investigation of the Effect of Different Remineralizing Agents on Human Enamel
}

\author{
Vincenzo Tosco, Riccardo Monterubbianesi, Scilla Sparabombe, Giulia Orilisi, Angelo Putignano and Giovanna \\ Orsini*
}

Polytechnic University of Marche, Department of Clinical Sciences and Stomatology (DISCO), Italy

*Corresponding author: Giovanna Orsini, Polytechnic University of Marche, Department of Clinical Sciences and Stomatology

(DISCO), Via Tronto 10, 60126, Ancona, Italy

\begin{abstract}
This in vitro study aimed to qualitatively evaluate the effect of different remineralizing agents on the enamel pattern, after exposure to an acid solution. Fifteen human sound extracted third molars were collected and randomly allocated into the following five groups: Control Group (CG), without treatment; Negative Group (NG), treated with lactic acid solution; Mousse Group (MG), treated using a mousse of casein phosphopeptide (CPP) and amorphous calcium phosphate (ACP), associated with $0,33 \%$ sodium monofluorophosphate; Biosmalto Group (BG), treated with a dentifrice containing amorphous calcium phosphate functionalized with fluorine and carbonate-coated with citrate; Duraphat Group (DG), treated with a 5\% sodium fluoride varnish. While CG remained immersed in chloramine solution for the entire period of the study, all the other groups were immersed in lactic acid for 2 hours and then subjected to the appropriate treatment for additional 2 hours, repeating the protocol 3 times a day, for 8 days. Finally, all samples were examined by Scanning Electron Microscope (SEM). SEM micrographs showed that CG had the typical morphological features of sound enamel, whereas NG presented superficial irregularities with early pattern of demineralization, characterized by apparent destruction of enamel prism cores. MG showed intact enamel prismatic arrangement and slight interprismatic dissolution, while BG and DG presented not defined margins of enamel prims and partial loss of interprismatic substance, being more pronounced in DG. Qualitative SEM evaluations demonstrate that topical applications of CPP+ACP associated with sodium monofluorophosphate could represent the most effective protocol for re-establishing the intact enamel structure, given acid environment.
\end{abstract}

Keywords: Dental caries; Dental enamel; CPP-ACP; Fluoride varnish; Sodium fluoride; Tooth demineralization; Tooth remineralization

\section{Introduction}

Dental caries remains a fundamental concern of today's practice and continues to be one of the most common diseases in the world, despite the significant progress of research and the diffused home-practice of oral hygiene [1]. The current concept of the caries forming process is based on the accumulation of numerous episodes of demineralization and remineralization, initiated by acid-producing bacteria in the oral micro-environment [2,3]. The demineralization of dental hard tissue still represents a growing problem and appears to be the main responsible for dentinal hypersensitivity and dental caries insurgence. Demineralization occurs before cavitation when there is a loss of minerals from hard dental tissues. Conversely, remineralization occurs when the calcium and phosphate gradients invert and spread towards inside of the lesion [4]. Natural remineralization in the initial phase is stimulated by saliva, which contains calcium ions, phosphate ions, buffer agents, fluoride and other substances [5]. However, when this ideal condition is lacking, topical agents become essential to avoid the demineralization. Nowadays, the topical fluoride plays a crucial role for caries prevention and for remineralization of the dental structure. The presence of fluoride ions in the oral cavity causes the fluorapatite to precipitate the calcium and phosphate ions existing in the saliva. The increase in $\mathrm{pH}$ will therefore lead to the formation of larger crystals resistant to fluorine-containing acids (fluorohydroxyapatite), developing a strong surface layer which increases the resistance to demineralization [6]. However, products containing calcium, phosphate and fluoride in their bio-available 
forms have claimed to increase remineralization over products containing only fluoride [5]. Among these, the remineralizing agent which has been extensively studied and accepted is casein phosphopeptide-amorphous calcium phosphate (CPP-ACP). CPPACP is a new agent for the prevention of dental caries [7]. This milk product facilitates remineralization, inhibits demineralization, and prevents dental caries by forming a calcium phosphate reservoir [8]. Indeed, casein phosphate that occurs in CPP-ACP stabilizes calcium and phosphate and facilitates the formation of calcium phosphate nano-complexes on the tooth surface. These compounds act as a source of minerals for the remineralization process. Subsequently, amorphous calcium phosphate forms and localizes on the tooth surface and acts as a source of calcium and phosphate ions. This material could also quickly turn into hydroxyapatite which is then deposited on the tooth surface $[9,10]$. Several studies affirm that CPP-ACP is anticariogenic and capable of reversing the early stages of enamel lesions in vitro and in clinical research [2]. Also, it is reported that CPP-ACP and flour combination (ACFP) create a synergic effect in caries prevention [11,12]. Therefore, the literature confirms that demineralization can be countered or reversed through different remineralization agents in non-cavitated carious lesions, although there is confusion and inaccuracy of their potential applications. Therefore, it is of great importance to study new strategies and to know different agents to improve the remineralization process. For this reason, the present in vitro study aims to qualitatively evaluate, by means of scanning electron microscopy (SEM), the effect of different remineralizing agents on enamel morphology after exposure to an acid solution.

The following null hypotheses were tested:

a. The remineralizing agents do not influence the demineralized enamel surface after acid environment exposure and

b. There is not difference on the effect of the three remineralizing agents.

\section{Materials and Methods}

Fifteen sound third molars were extracted and collected for the present study. The exclusion criteria consisted of presence of lesions and decays, including white spots or hypomineralized lesions, fracture of either crown or root, developmental defects or any other crown deformities. After the surgical extraction, the soft tissue debris were cleaned using an ultrasonic scaler with distilled water. After that, the samples were cut horizontally using a diamond disk coupled to a handpiece, sectioning the crown from the root, and then stored in a $0,5 \%(\mathrm{w} / \mathrm{w})$ chloramine solution $(\mathrm{NH} 2 \mathrm{Cl})$. Afterwards, the samples were randomly assigned into the following five groups $(n=3)$ : Control Group (CG), without treatment; Negative Group (NG), treated with demineralizing solution ( $\mathrm{pH}=4.4)$; Mousse Group (MG), treated using GC Tooth Mousse (Recaldent, GC Europe) a mousse of casein phosphopeptide (CPP) and amorphous calcium phosphate (ACP), associated with 0,33\% sodium monofluorophosphate; Biosmalto Group (BG), treated with Biosmalto (Curasept S.p.A., Italy), a mousse containing amorphous calcium phosphate functionalized with fluorine and carbonate-coated with citrate; Duraphat Group (DG), treated with Duraphat (Colgate-Palmolive), a $5 \%$ sodium fluoride varnish. While CG remained immersed in chloramine solution for the entire period of the study, changing the chloramine solution every 4 days, all the other groups were immersed in a demineralizing solution containing a $0.1 \mathrm{M}$ acid lactic, adjusted to $\mathrm{pH} 4.4$, for 2 hours [13], and then subjected to the appropriate treatment for additional 2 hours, repeating the protocol 3 times a day, for 8 days. Finally, after treatment procedures, all samples were carefully cleaned, dehydrated and gold sputter-coated for evaluation using a SEM (Scanning Electron Microscope Zeiss Supra 40, Department of SIMAU, Polytechnic University of Marche, Italy), operated at $30 \mathrm{kV}$ and at a working distance of $12 \mathrm{~mm}$. Five pictures for each sample at different magnification were performed (4000x and 10000x).

\section{Results}

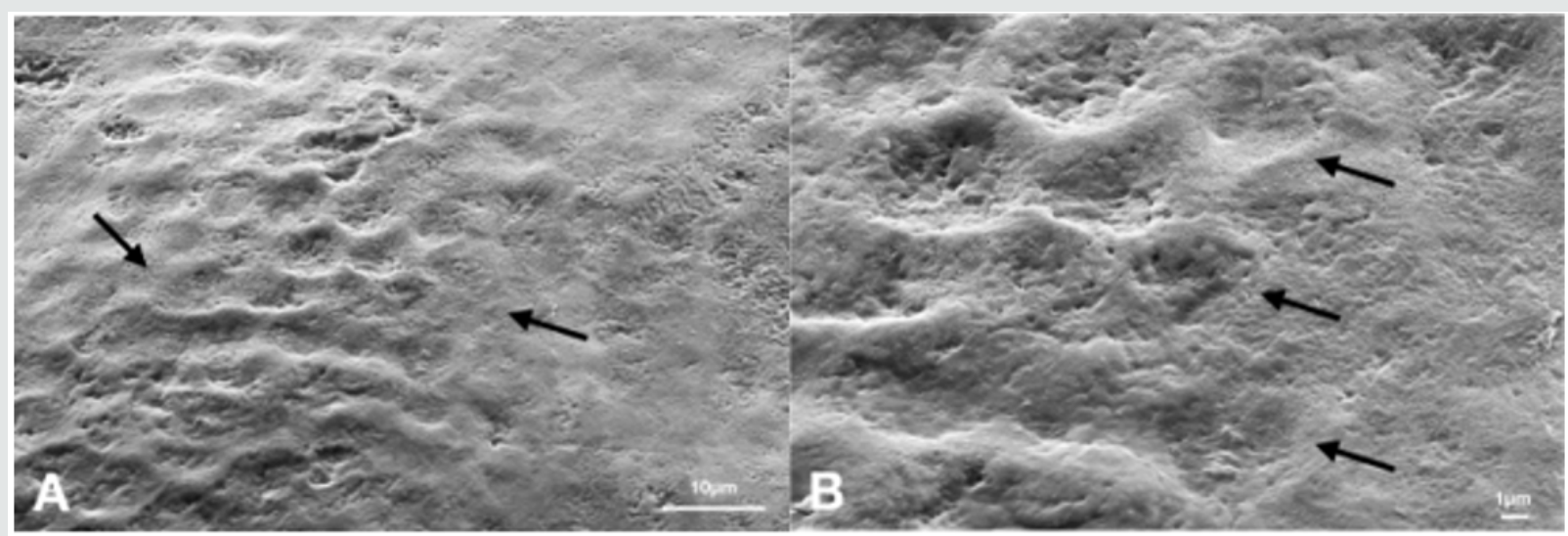

Figure 1: Scanning electron microphotographs of a representative CG Group tooth sample at (A) 4.0K, and (B) 10.00K original magnification. In (A), the typical aspect of sound enamel morphology, with enamel-like crystal structure (black arrows) is showed. In (B), a homogeneous smoothed area between the interprismatic rods (black arrows) is highlighted. 
Scanning electron micrographs, taken at different magnification in the 5 groups, showed dissimilar morphological features of the enamel surface, as follows.

CG displayed the characteristic sound enamel morphology with intact enamel rods and interrods (Figure 1), while NG presented an irregular surface with early pattern of demineralization, characterized by interprismatic tissue dissolution without apparent destruction of enamel prism cores but retained periphery (Figure 2). MG showed a prismatic disposition of the native enamel and a slight interprismatic dissolution. A newly-formed layer of needlelike crystals has been deposited on the surface of the demineralized enamel. These crystals seemed to be randomly arranged, parallel to the surface, reconstructing a structure which is like the intact enamel unit (Figure 3). BG showed an incomplete newly-formed layer of needle-like crystals. The disposition of these latter crystals was rather irregular, thus presenting a partial prisms dissolution which does slightly resemble the native enamel unit (Figure 4). Finally, the DG presented undefined margins of enamel prisms and partial loss of interpretismatic substance, displaying several areas of disorganized arrangement. Therefore, DG results show a partially demineralized surface with no evident signs of reorganization of the native structure of the sound enamel (Figure 5).

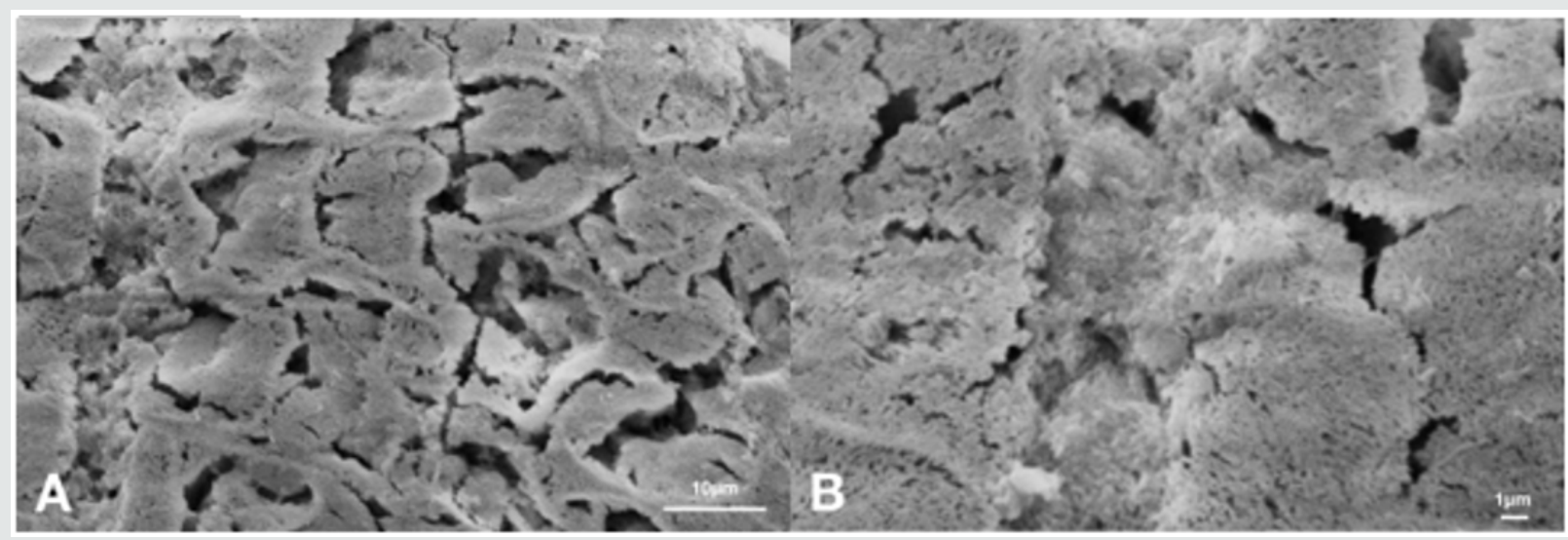

Figure 2: Scanning electron microphotographs of a representative NG Group tooth sample at (A) 4.0K, and (B) $10.00 \mathrm{~K}$ original magnification. The typical aspect of enamel demineralization. After exposed to the acid lactic solution, the enamel morphology presents an early stage of prisms destruction, with slight interprismatic tissue dissolution occurred at the peripheral areas of the prisms. The margins of the prisms are not clearly identifiable.

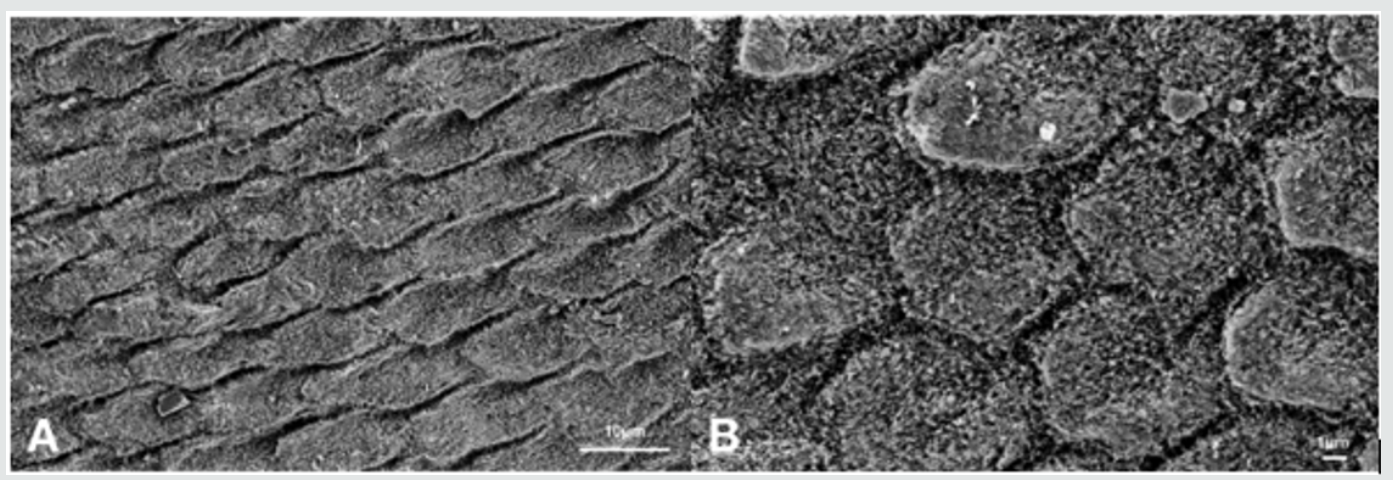

Figure 3: Scanning electron microphotographs of a representative MG Group tooth sample at (A) $4.0 \mathrm{~K}$, and (B) $10.00 \mathrm{~K}$ original magnification. The intact enamel prismatic arrangement and slight interprismatic dissolution. Presence of the ideal oriented and ordered mineralization pattern partially accomplished, with the formation of mineral crystals parallel on enamel surface.

\section{Discussion}

The understanding of the imbalance between demineralization and remineralization process is the key factor for caries control and prevention $[4,14]$. Several studies confirm that an effective management of carious lesions is to early detect the initial demineralization and to use non-invasive techniques by means of various remineralizing agents [15]. A lot of products present in the market, containing calcium, phosphate and fluoride in their bioavailable forms, have claimed to develop and increase the remineralization process. The objective of this in vitro study was to qualitatively evaluate the remineralization effect of three different agents on the enamel surface after exposure of acid environment: GC Tooth Mousse+sodium monofluorophosphate, Biosmalto mousse and Duraphat varnish. the results of this study rejected both of the null hypothesis that the remineralizing agents does not effect the demineralized enamel surface after acid environment exposure and there is not different between on the effect of three remineralizing agents. Since it is difficult to reproduce the demineralization phase in the oral environment in 
vitro, several studies have developed different demineralization methods using $\mathrm{pH}$ cycle and acid-etched models, to obtain an artificial enamel lesion [3,16-18]. This study, in agreement with the report of Bonetti et al., shows that an exposure under $0.1 \mathrm{M}$ acid lactic ( $\mathrm{pH} 4.4)$ can reproduce the effects of the demineralization occurring on the enamel surface, thus simulating the initial step of a carious lesion [13]. In fact, the scanning electron micrographs of NG demonstrate an early pattern of demineralization with evidence of interprismatic tissue loss. In particular, the demineralized enamel surface exhibited a loss of the enamel prism core, without preserving the periphery, leading to a disordered arrangement of the rod-like residual [19]. Therefore, to oppose these effects, a lot of products present on the market, containing calcium, phosphate, synthetic hydroxyapatite and fluoride in their bioavailable forms, have been claimed to enhance the remineralization process [2022]. The null hypotheses of the present qualitative evaluation on the effects of the tested three commonly used remineralizing agents, on enamel surface, after exposure of acid environment, were both rejected. Indeed, MG, BG and DG do influence the superficial enamel demineralization and there is a difference among the effects exerted by the three products. MG (CPP-ACP associated with $0,33 \%$ of sodium monofluorophosphate) showed the most promising results. Indeed, SEM images of MG seem to reveal an attempt to recover tissues at the level of the superficial enamel layers, with a predominantly intact and regular interprismatic structure (Figure 3). Moreover, the electron scanning observations displayed the formation of oriented and ordered mineral crystals parallel to the enamel surface. Although ideal oriented and ordered mineralization pattern was accomplished partially, it is noteworthy that the creation of these mineral crystals parallel on enamel surface could be attributed to a secondary crystallization [19]. This suggests an excellent remineralizing potential of the MG, also confirmed by several studies, affirming that, given acid environmental conditions, topical applications of CPP-ACP can be effective in increasing enamel remineralization [13,23]. Indeed, CPP-ACP complex, a milk protein derivative, has been proven to have anticariogenic effects [24-26]. Some studies report that its association with fluoride (CPPACPF) improves remineralization [6,27]. Numerous authors have shown that there was a consistent increase in remineralization and a decrease in carious lesion depth when combining the latter two $[12,28,29]$. Jayarajan et al. [5] presented that the added value of fluoride (NaF $0.2 \%$ ) to CPP-ACP slightly showed more amount of remineralization than CPP-ACP alone. Therefore, the results analyzed in this study confirm the potential benefits of CPP-ACPF, as frequently highlighted by the scientific literature $[17,20,24,28]$.

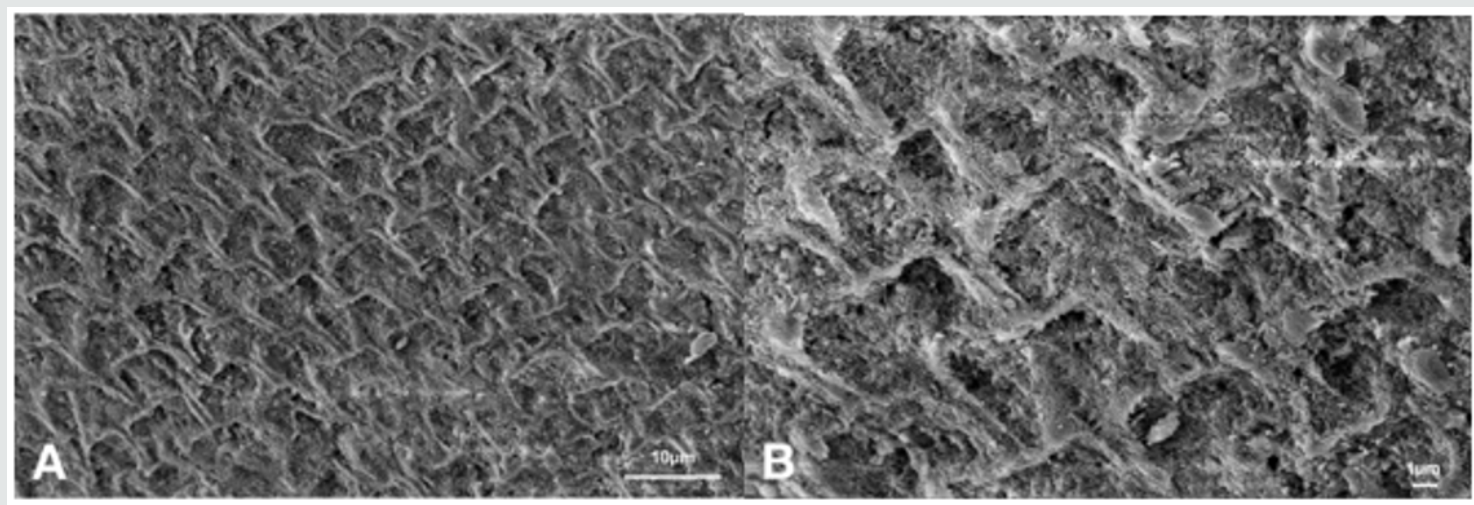

Figure 4: Scanning electron microphotographs of a representative BG Group tooth sample at (A) $4.0 \mathrm{~K}$, and (B) $10.00 \mathrm{~K}$ original magnification. The honeycomb structure was at an early stage, with slight prism cores dissolution. The enamel-like crystal structure reminds a potential remineralizing of the substrate. The new crystal formed on the surface of the demineralized enamel is not evident.

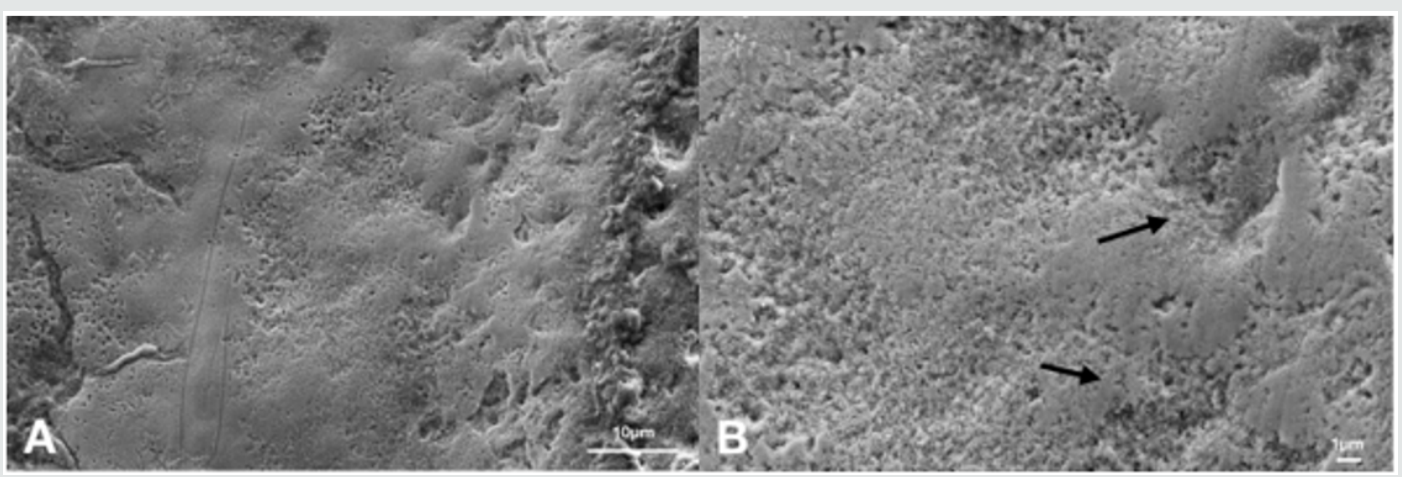

Figure 5: Scanning electron microphotographs of a representative DG Group tooth sample at (A) 4.0K, and (B) 10.00K original magnification. The predominant irregular structure, with slight prism cores dissolution. The margins of the interprismatic structure of the enamel are not evident, presenting a surface disorganization (black arrows). 
Regarding BG group, it is possible to observe the presence of a partially interprismatic dissolution of the enamel surface. Several studies demonstrated that citrate ions play an important role in stabilizing the precursor ACP phase [30-32]. In addition, [33], showed that the use of fluoride and citrate modify specific ACP and ACPF physico-chemical properties, to generate potentially anti-caries agents and remineralizing effects [33]. Furthermore, by adding citrate to these fluoride-doped ACP nanoparticles, it is possible to restore enamel in its natural structure [31]. ACPFcitrate treated samples showed a faster remineralization effect compared to the ACP- treated ones, especially at the level of enamel prismatic structure, thus being suitable for patients exhibiting severe demineralization [33]. Indeed, in partial disagreement with this latter beneficial effect report, our finding does not clearly demonstrate improved remineralization in BG (Figure 4). Although the newly deposited crystals are less ordered than the natural ones and rather incomplete, it is remarkable that they form a layer of enamel-like crystals, mimicking enamel substrate. In this sense, it would seem possible that the remineralizing action of ACPF combined with citrate is less pronounced than the one exhibited by CCP-ACP associated with $0,33 \%$ sodium monofluorophosphate of MG. Among the groups treated with the three remineralizing compounds, DG, in which a $5 \%$ sodium fluoride-based varnish was applied, seems to be the one with less evident remineralizing benefits on the superficial enamel, partially confirmed to what is reported by the existing scientific literature [34,35]. Although several studies have assessed the beneficial effects of fluoride varnishes on the remineralization process, Duraphat appears to be the least effective [36-38]. For instance, to prevent the incipient caries, [34] concluded that the varnish containing 5\% sodium fluoride associated with CPP-ACP was shown to be more effective than the conventional one based on 5\% sodium fluoride [34]. In addition, these data are consistent with [35] that, analyzing three different varnishes, obtained an increase of enamel surface microhardness in samples treated with varnish containing $5 \%$ sodium fluoride associated with CPP-ACP [35]. In disagreement with these latter reports and with our findings, some studies claimed that $5 \%$ sodium fluoride varnish represents the most effective agent for remineralization $[39,40]$. Therefore, due to the controversial scientific evidence, the clear and effective advantages of fluoride-based varnishes are still debated, and caution must be paid interpreting the results of the present studies. Scanning electron micrographs obtained by DG displayed a predominantly uneven surface. After the remineralization treatment, several areas of demineralized enamel are still evident, showing a loss of interprismatic substance and disorganized arrangement. Therefore, the attempt to form an ideally-oriented and ordered mineralization pattern on enamel surface has not occurred. The current study design was limited by the in vitro simulation of oral cavity conditions. Another limitation is that Scanning Electron Micrographs do not allow to clearly evaluate the real potential of these products, since only qualitative observations have been obtained. Further studies are needed to quantitively evaluate the changes after treatment, by determining the chemical composition of enamel structure and performing micro-hardness test.

\section{Conclusion}

Considering the limitations of this in vitro study, it can be concluded that, in the event of exposure to acid environmental conditions, topical applications of CPP+ACP associated with $0,33 \%$ of sodium monofluorophosphate may represent the most effective protocol for re-establishing the intact enamel structure.

\section{Acknowledgement}

This work was supported by funds of the Polytechnic University of Marche. The authors would like to thank Adriano Di Cristoforo, Department of Materials, Environmental Science and Urban Planning (SIMAU), CISMIN, Polytechnic University of Marche. He contributed to the setting of Scanning Electron Microscopy for enamel surface analysis. Also, would like to thank Maria Cristina Storaci for samples preparation.

\section{Conflicts of Interest}

The authors certify that there is no conflict of interest regarding the publication and no financial relationships relevant to this article.

\section{Author Contributions}

V. Tosco contributed to the acquisition and interpretation of data and manuscript discussion. R. Monterubbianesi and S. Sparabombe contributed to the study design and data interpretation. G. Orilisi performed the SEM analysis and contributed to data interpretation. A. Putignano and G. Orsini contributed to the research plan, data interpretation and supervised the entire project. All authors participated to the writing of the present manuscript.

\section{References}

1. Madhu Wagle, Francesco D Antonio, Eirik Reierth, Purusotam Basnet, Tordis A Trovik, et al. (2018) Dental caries and preterm birth: A systematic review and meta-analysis. BMJ Open 8(3): e018556.

2. Ma X, Lin X, Zhong T, Xie F (2019) Evaluation of the efficacy of casein phosphopeptide-amorphous calcium phosphate on remineralization of white spot lesions in vitro and clinical research: A systematic review and meta-analysis. BMC Oral Health 19(1): 295.

3. Thimmaiah C, Shetty P, Shetty SB, Natarajan S, Thomas NA (2019) Comparative analysis of the remineralization potential of CPP-ACP with Fluoride, Tri-Calcium Phosphate and Nano Hydroxyapatite using SEM/EDX - An in vitro study. J Clin Exp Dent 11(12): 1120-1126.

4. Neel EAA, Aljabo A, Strange A, Ibrahim S, Coathup M, et al. (2016) Demineralization \& remineralization dynamics in teeth and bone. International Journal of Nanomedicine 11: 4743-4763.

5. Jayarajan J, Janardhanam P, Jayakumar P, Deepika (2011) Efficacy of CPPACP and CPP-ACPF on enamel remineralization-An in vitro study using scanning electron microscope and DIAGNOdent. Indian Journal of Dental Research 22(1): 77-82.

6. Lata S, Varghese NO, Varughese JM (2010) Remineralization potential of fluoride and amorphous calcium phosphate-casein phospho peptide on enamel lesions: An in vitro comparative evaluation. J Conserv Dent 13: $42-46$

7. DALOĞLU M, Ulu Güzel KG (2018) Remineralizing Agents: Review. Turkiye Klinikleri Journal of Dental Sciences 24.

8. Memarpour M, Fakhraei E, Dadaein S, Vossoughi M (2015) Efficacy of Fluoride Varnish and Casein Phosphopeptide-Amorphous Calcium Phosphate for Remineralization of Primary Teeth: A Randomized Clinical Trial. Med Princ Pract 24(3): 231-237. 
9. Rahiotis C, Vougiouklakis G (2007) Effect of a CPP-ACP agent on the demineralization and remineralization of dentine in vitro. J Dent 35(8): 695-698.

10. Ranjitkar S, Narayana T, Kaidonis JA, Hughes TE, Richards LC, et al. (2009) The effect of casein phosphopeptide-amorphous calcium phosphate on erosive dentine wear. Australian Dental Journal 54(2): 101-107.

11. Manoharan V, Kumar RK, Sivanraj AK, Arumugam SB (2018) Comparative Evaluation of Remineralization Potential of Casein Phosphopeptide-Amorphous Calcium Fluoride Phosphate and Novamin on Artificially Demineralized Human Enamel: An In vitro Study. Contemp Clin Dent 9: S58-S63.

12. Imani MM, Safaei M, Afnaniesfandabad A, Moradpoor H, Sadeghi M, et al. (2019) Efficacy of CPP-ACP and CPP-ACPF for Prevention and Remineralization of White Spot Lesions in Orthodontic Patients: A Systematic Review of Randomized Controlled Clinical Trials. Acta Inform Med 27(3): 199-204.

13. Giulio AB, Matteo Z, Serena IP, Silvia M, Luigi C (2009) In vitro evaluation of casein phosphopeptide-amorphous calcium phosphate (CPP-ACP) effect on stripped enamel surfaces. A SEM investigation. Journal of Dentistry 37(3): 228-232.

14. Zhang X, Li Y, Sun X, Kishen A, Deng X, et al. (2014) Biomimetic remineralization of demineralized enamel with nano-complexes of phosphorylated chitosan and amorphous calcium phosphate. J Mater Sci Mater Med 25(12): 2619-2628.

15. Gangrade A, Gade V, Patil S, Gade J, Chandhok D, et al. (2016) In vitro evaluation of remineralization efficacy of different calcium- and fluoride-based delivery systems on artificially demineralized enamel surface. J Conserv Dent 19(4): 328-331.

16. Featherstone JDB, Barrett Vespone NA, Fried D, Kantorowitz Z, Seka W (1998) $\mathrm{CO}_{2}$ Laser Inhibition of Artificial Caries-like Lesion Progression in Dental Enamel. J Dent Res 77(6): 1397-1403.

17. Singhal RK, Rai B (2017) Remineralization Potential of Three Toothpastes on Enamel Caries. Open Access Maced J Med Sci 5(5): 664-666.

18. Yang Y, Lv X, Shi W, Zhou X, Li J, et al. (2016) Synergistic Inhibition of Enamel Demineralization by Peptide 8DSS and Fluoride. Caries Res 50(1): 32-39.

19. Wang H, Xiao Z, Yang J, Lu D, Kishen A, et al. (2017) Oriented and Ordered Biomimetic Remineralization of the Surface of Demineralized Dental Enamel Using HAP@ACP Nanoparticles Guided by Glycine. Scientific Reports 7.

20. Carrouel F, Viennot S, Ottolenghi L, Gaillard C, Bourgeois D (2020) Nanoparticles as Anti-Microbial, Anti-Inflammatory, and Remineralizing Agents in Oral Care Cosmetics: A Review of the Current Situation. Nanomaterials (Basel) 10(1): 140.

21. Orsini G, Procaccini M, Manzoli L, Sparabombe S, Tiriduzzi P, et al. (2013) A 3-day randomized clinical trial to investigate the desensitizing properties of three dentifrices. J Periodontol 84(11): e65-73.

22. Lelli M, Putignano A, Marchetti M, Foltran I, Mangani F, et al. (2014) Remineralization and repair of enamel surface by biomimetic Zn-carbonate hydroxyapatite containing toothpaste: A comparative in vivo study. Front Physiol 5: 333.

23. Sinfiteli P de P, Coutinho TCL, Oliveira PRA de, Vasques WF, Azevedo LM, et al. (2017) Effect of fluoride dentifrice and casein phosphopeptide-amorphous calcium phosphate cream with and without fluoride in preventing enamel demineralization in a $\mathrm{pH}$ cyclic study. Journal of Applied Oral Science 25(6): 604-611.

24. Reynolds EC, Cai F, Shen P, Walker GD (2016) Retention in Plaque and Remineralization of Enamel Lesions by Various Forms of Calcium in a Mouthrinse or Sugar-free Chewing Gum. Journal of Dental Research 82(3): 206-11.
25. Mazzaoui SA, Burrow MF, Tyas MJ, Dashper SG, Eakins D, et al. (2016) Incorporation of Casein Phosphopeptide-Amorphous Calcium Phosphate into a Glass-ionomer Cement. Journal of Dental Research 27(3): 235-43.

26. Kumar VLN, Itthagarun A, King NM (2008) The effect of casein phosphopeptide-amorphous calcium phosphate on remineralization of artificial caries-like lesions: an in vitro study. Australian Dental Journal 53(1): 34-40.

27. Llena C, Forner L, Baca P (2009) Anticariogenicity of casein phosphopeptide-amorphous calcium phosphate: A review of the literature. J Contemp Dent Pract 10(3): 1-9.

28. Mielczarek A, Gedrange T, Michalik J (2015) An in vitro evaluation of the effect of fluoride products on white spot lesion remineralization. Am J Dent 28(1): 51-56.

29. Reema SD, Lahiri PK, Roy SS (2014) Review of casein phosphopeptides-amorphous calcium phosphate. Chin J Dent Res: 17(1): 7-14.

30. Ivanchenko P, Delgado López JM, Iafisco M, Gómez Morales J, Tampieri A, et al. (2017) On the surface effects of citrates on nano-apatite's: Evidence of a decreased hydrophilicity. Sci Rep 7: 1-10.

31. Delgado López JM, Iafisco M, Rodríguez I, Tampieri A, Prat M, et al. (2012) Crystallization of bioinspired citrate-functionalized nanoapatite with tailored carbonate content. Acta Biomater 8(9): 3491-3499.

32. José Manuel Delgado-López, Ruggero Frison, Antonio Cervellino, Jaime Gómez-Morales, Antonietta Guagliardi, et al. (2014) Crystal Size, Morphology, and Growth Mechanism in Bio-Inspired Apatite Nanocrystals. Advanced Functional Materials-Wiley Online Library 24(8): 1090-1099.

33. Iafisco M, Degli Esposti L, Ramírez-Rodríguez GB, Carella F, Gómez-Morales J, et al. (2018) Fluoride-doped amorphous calcium phosphate nanoparticles as a promising biomimetic material for dental remineralization. Sci Rep 8.

34. Pithon MM, Dos Santos MJ, Andrade CSS, Leão Filho JCB, Braz AKS, et al. (2015) Effectiveness of varnish with CPP-ACP in prevention of caries lesions around orthodontic brackets: an OCT evaluation. Eur J Orthod 37(2): 177-182.

35. Kooshki F, Pajoohan S, Kamareh S (2019) Effects of treatment with three types of varnish remineralizing agents on the microhardness of demineralized enamel surface. Journal of Clinical and Experimental Dentistry 11(7): 630-635.

36. Molaasadolah F, Eskandarion S, Ehsani A, Sanginan M (2017) In Vitro Evaluation of Enamel Microhardness after Application of Two Types of Fluoride Varnish. J Clin Diagn Res 11(8): ZC64-ZC66.

37. Almeida M, Costa O, Ferreira J, Menezes V, Leal R, et al. (2011) Therapeutic potential of Brazilian fluoride varnishes: An in vivo study. Brazilian Dental Journal 22(3): 193-197.

38. Ferreira JMS, Aragão AKR, Rosa ADB, Sampaio FC, Menezes VA de (2009) Therapeutic effect of two fluoride varnishes on white spot lesions: A randomized clinical trial. Braz Oral Res 23(4): 446-451.

39. Slayton RL, Urquhart O, Araujo MWB, Fontana M, Guzmán Armstrong S, et al. (2018) Evidence-based clinical practice guideline on nonrestorative treatments for carious lesions: A report from the American Dental Association. The Journal of the American Dental Association 149(10): 837-849.e19.

40. Fernández Ferrer L, Vicente Ruíz M, García Sanz V, Montiel Company JM, Paredes Gallardo V, et al. (2018) Enamel remineralization therapies for treating postorthodontic white-spot lesions: A systematic review. The Journal of the American Dental Association 149(9): 778-786.e2. 
(c) (i) This work is licensed under Creative

To Submit Your Article Click Here: Submit Artide

DOI: 10.32474/MADOHC.2020.04.000189

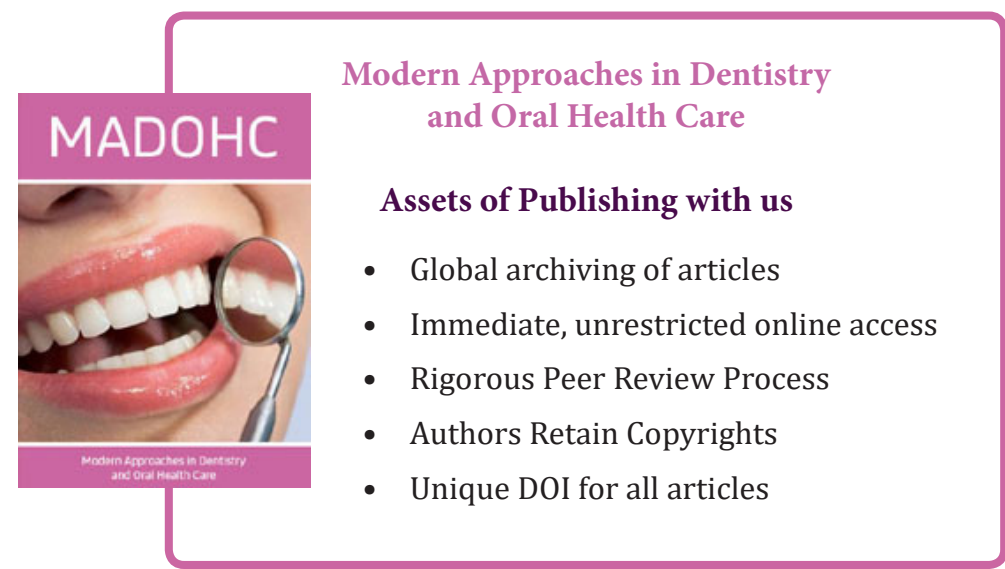

